\title{
THEORETICAL AND EXPERIMENTAL STUDIES OF CIRCULATIONS INSIDE AND OUTSIDE A DEFORMED DROP UNDER A UNIFORM ELECTRIC FIELD
}

\author{
TAKAO TSUKADA \\ Department of Biochemistry and Engineering, Tohoku University, \\ Sendai 980 \\ TETSUYA KATAYAMA, YUKIKAZU ITO \\ AND MITSUNORI HOZAWA* \\ Institute for Chemical Reaction Science, Tohoku University, \\ Sendai 980
}

Key Words: Drop Deformation, Electrohydrodynamics, Electrostatics, Numerical Simulation, Finite Element Method

\begin{abstract}
A finite element computational technique has been developed for exactly calculating circulations inside and outside a dielectric drop suspended in another dielectric fluid under a uniform electric field. This technique allows the prediction of not only the circulations but also drop deformation caused by electric and dynamic forces acting over the drop surface. Computed results show that the direction of the circulations and the drop deformation depend on physical properties of the fluids, especially the drop-to-medium ratios of electric conductivity and dielectric constant. These results are in good agreement with Taylor's analytical solutions and authors' experimental observations.
\end{abstract}

\section{Introduction}

When a uniform electric field is applied to a dielectric fluid in which a drop of another dielectric fluid is suspended, circulations are induced both inside and outside the drop. They are ascribable to a tangential stress resulting from an interaction between the electric field and a charge built-up at the drop/continuous phase interface. This electric interaction yields a normal stress as well, which contributes to drop deformation against the interfacial tension working at the drop surface. The circulations are of practical interest since they possibly enhance heat and mass transfer between the drop and the continuous phase. Thus extensive studies have been carried out from the viewpoint of improving the efficiencies of directcontact heat and mass exchangers with the aid of such circulations. A brief survey of the literature is given below.

Taylor ${ }^{7)}$ considered a stationary spherical drop of a leaky dielectric fluid in another dielectric fluid under a uniform D.C. electric field, and analyzed the circulations generated inside and outside the drop by the electric field. From the analysis, he proposed the following criteria for drop deformation.

$$
\begin{aligned}
& \Psi<0: \text { oblate } \\
& \Psi=0: \text { spherical } \\
& \Psi>0: \text { prolate }
\end{aligned}
$$

where $\Psi$ is a parameter composed only of some physical properties of the two fluids. It is defined as

$$
\begin{aligned}
& \Psi(R, S, \kappa)=S /(R+2)^{2} \\
& \quad \times\left\{\left(R^{2}+1\right) / S-2+3(R / S-1)(3 \kappa+2) /(5 \kappa+5)\right\}
\end{aligned}
$$

\footnotetext{
* Received April 20, 1993. Correspondence concerning this article should be addressed to M. Hozawa.
}

In Eq. (2), $R$ is the electric conductivity ratio, $S$ is the permittivity ratio and $\kappa$ is the viscosity ratio of the drop to the surroundings. Taylor ${ }^{7)}$ also showed criteria for the flow direction at the interface: when $R<S$, the flow direction is from the pole to the equator, and when $R>S$, it is opposite. Torza et al. ${ }^{8)}$ developed a theory which is valid for both steady and alternating electric fields and is reduced to Taylor's theory in the steady field, and compared it with their experimental observations. According to their analysis, the following expression for steady drop deformation can be derived.

$$
a / b=\left(1+D_{\omega}\right) /\left(1-D_{\omega}\right)
$$

where $a$ and $b$ are vertical and horizontal axes of the deformed drop,

$$
D_{\omega}=(9 / 128 \pi) \Psi_{\omega} E_{0}{ }^{* 2}
$$

and $\Psi_{\omega}$ is a parameter involving the frequency of the electric field, $\omega$, and is reduced to Eq. (2) when $\omega=0$. In this theory the drop is assumed to be nearly spherical, so that the theory gives a first-order approximation of the deformation.

When a uniform electric field is applied to a dielectric drop moving under the influence of gravity, the effect of the electric field is coupled with that of gravity, and hence the flow configuration around the drop becomes more complicated. Chang et al. ${ }^{1,2)}$ computed the flow pattern around a drop translating parallel to the electric field at a small or intermediate Reynolds number. They ${ }^{3)}$ also investigated theoretically the effect of the presence of a surfactant on the flow pattern around 


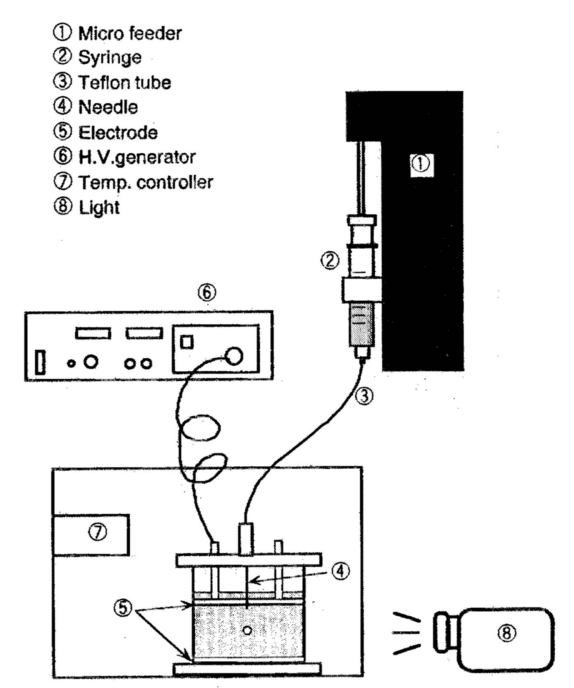

Fig. 1 Experimental apparatus

the drop in the electric field.

In addition, there have been a few theoretical studies on the relationship between the flow pattern around stationary drops ${ }^{4,6)}$ or translating drops ${ }^{1,2)}$ and the mass and heat transfer rates through the drop surface under electric fields. For instance, Chang et al. ${ }^{1,2)} \mathrm{com}-$ puted the effect of electric field strength on the mass transfer coefficient. However, there have been few experimental studies that directly demonstrate the above effect, i.e. the effect of the Taylor circulations on heat and mass transfer.

All of the previous mathematical models for electrically induced circulations are valid only for spherical or nearly spherical drops. However, each drop can be deformed by the electric and dynamic forces acting on its surface, and the deformation becomes more significant as the electric field strength increases.

The aim of the present work is to develop a mathematical model that can predict exactly the drop shape together with the flow field when a dielectric drop is stationarily suspended in another immiscible, dielectric liquid under a uniform electric field, and to investigate theoretically the effect of the electric field on the circulations inside and outside the deformed drop. Theoretical studies of the flow field and the drop shape for translating drops under the influence of an electric field will be reported elsewhere.

\section{Experiment}

Figure 1 shows schematically the experimental apparatus used in the present work. It essentially consists of a cell equipped with two electrodes, a drop formation device, and a high-voltage regulated D.C. power supply connected to one of the electrodes. A drop with a given volume was supplied into the continuous liquid through an injection needle from a syringe mounted on a microfeeder. Then a high D.C. voltage was applied to the
Table 1. Physical Properties (at 303 K)

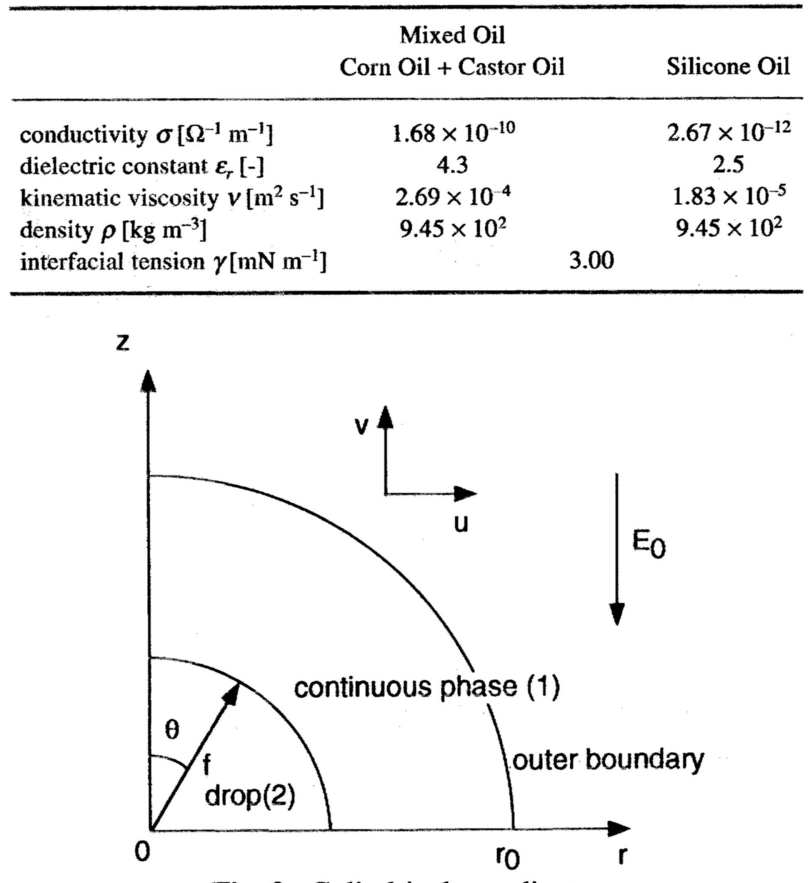

Fig. 2 Cylindrical coordinates

system. For measurements of drop shape and drop volume, photographs of the drop were taken using a back-light source. Visualization of flow patterns around the drop was carried out, using micro-capsules of a liquid crystal as tracer particles. The cell was made of transparent acrylic resin. It held a pair of parallel copper electrodes $(100 \times 100 \mathrm{~mm})$. The distance between the electrodes was $50 \mathrm{~mm}$, and D.C. electric fields in the range of 0-20,000 V were applied across it. Drop diameter was in the range of 4.7-5.8 $\mathrm{mm}$. The whole experimental system was set in an air bath controlled at $303 \mathrm{~K}$.

A silicone oil and a vegetable-oil mixture were used as dielectric, mutually immiscible liquids ${ }^{7)}$. To make each drop suspended stationarily in the continuous phase, a vegetable-oil mixture was prepared by mixing castor oil and corn oil at the volume ratio 190:610 so that its density well approximated that of the silicone oil. As a result, each drop of silicone oil was stationarily held in the vegetable-oil mixture for about 15 minutes. Physical properties of the silicone oil and the vegetable-oil mixture are listed in Table 1. Since it is difficult to measure the interfacial tension between two liquids having almost the same densities, a new technique for interfacial tension measurement developed by Karri et al. ${ }^{5)}$ was used.

\section{Theory}

2.1 Governing equations and boundary conditions

Figure 2 shows the cylindrical coordinates system used in calculations, where the behavior of a drop of fluid 2 surrounded by fluid 1 under the influence of a uniform electric field $E_{0}$ parallel to z-axis is considered. We assume that the densities of the two fluids are iden- 
tical, i.e. the effect of gravity is negligible. Hence, only the upper half of the drop is analyzed, as shown in Fig. 2. We also assume a steady state.

Since in a strict sense no fluid which is perfectly dielectric exists, a drop of a leaky dielectric fluid in another such fluid is considered here.

Under the above assumptions, the governing equations for the electric field, the equations describing the conservation of electric charge, are given as follows:

$$
\nabla \cdot j_{i}=0
$$

where the subscript $(i)$ represents the continuous phase ( $i$ $=1)$ or the drop $(i=2)$. By Ohm's law and the relationship between the electric field strength and the scalar potential $V$, Eq. (5) is rewritten as:

$$
\sigma_{i} \nabla^{2} V_{i}=0
$$

where $\sigma$ is the electric conductivity.

The boundary conditions for Eq. (6) are given by the following equations.

At the drop surface:

$$
\begin{gathered}
V_{1}=V_{2} \\
\sigma_{1}\left(\partial V_{1} / \partial n\right)=\sigma_{2}\left(\partial V_{2} / \partial n\right)
\end{gathered}
$$

In the field far from the drop:

$$
V_{1}=E_{0} r_{0} \cos \theta
$$

At the center line:

$$
\left(\partial V_{i} / \partial n\right)=0
$$

At the plane of symmetry:

$$
V_{i}=0
$$

The governing equations for the flow field, the momentum and continuity equations, are expressed as follows.

$$
\begin{gathered}
\rho_{i} \boldsymbol{v}_{i} \cdot \nabla \boldsymbol{v}_{i}=-\nabla p_{i}-\nabla \cdot \boldsymbol{\tau}_{i} \\
\nabla \cdot \boldsymbol{v}_{i}=0
\end{gathered}
$$

where $\tau$ is the stress tensor, defined as $-\mu\left(\nabla v+\nabla v^{T}\right)$.

These governing equations are subject to the following boundary conditions.

At the drop surface:

$$
\begin{gathered}
\boldsymbol{n} \cdot \boldsymbol{v}_{1}=\boldsymbol{n} \cdot \boldsymbol{v}_{2}=0 \\
\boldsymbol{\tau}_{1}: \boldsymbol{n} \boldsymbol{t}-\boldsymbol{\tau}_{2}: \boldsymbol{n} \boldsymbol{t}=\boldsymbol{\tau}_{2}^{E}: \boldsymbol{n} \boldsymbol{t}-\boldsymbol{\tau}_{1}^{E}: \boldsymbol{n t} \\
p_{1}-p_{2}+\boldsymbol{\tau}_{1}: \boldsymbol{n} \boldsymbol{n}-\boldsymbol{\tau}_{2}: \boldsymbol{n} \boldsymbol{n}+\boldsymbol{\tau}_{1}^{E}: \boldsymbol{n} \boldsymbol{n}-\boldsymbol{\tau}_{2}^{E}: \boldsymbol{n} \boldsymbol{n}=2 H \gamma
\end{gathered}
$$

In the field far from the drop:

$$
n \cdot v_{1}=\boldsymbol{t} \cdot \boldsymbol{v}_{1}=0
$$

At the center line and in the symmetric plane:

$$
\boldsymbol{n} \cdot \boldsymbol{v}_{i}=\boldsymbol{\tau}_{i}: \boldsymbol{n t}=\mathbf{0}
$$

Eq. (10-3) shows the tangential stress balance on the drop surface. Eq. (10-4) shows the normal stress balance

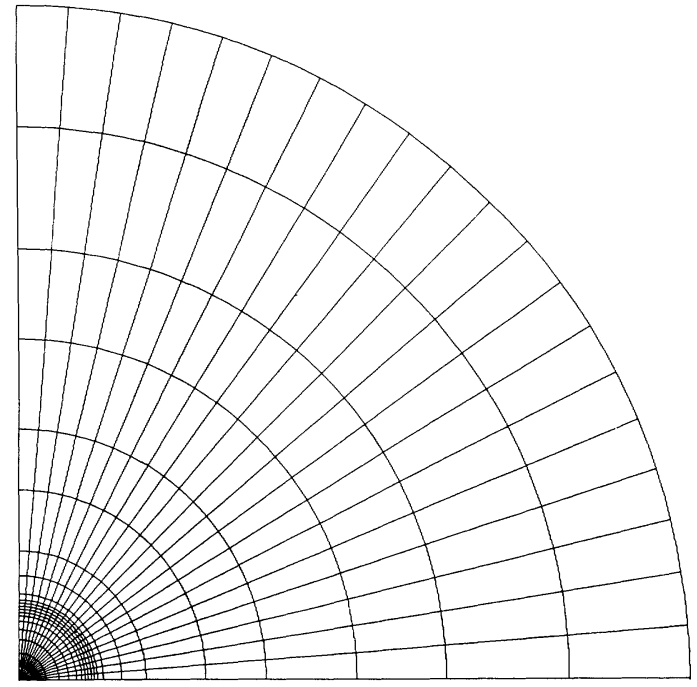

Fig. 3 Finite element discretization

that determines the drop shape. In Eqs. (10-3) and (104), $\tau^{E}$ is Maxwell's stress tensor, described by Eq.(11):

$$
\tau^{E}{ }_{l m}=-(\varepsilon / 2)|\boldsymbol{E}|^{2} \delta_{l m}+\varepsilon E_{l} E_{m}
$$

In addition to Eq. (10-4), the solution for drop shape should satisfy the constraint for a fixed volume of the drop.

$$
\int_{0}^{\frac{\pi}{2}} f^{3} \sin \theta d \theta=d_{e}^{3} / 8
$$

To evaluate the effect of the hydrodynamic stress on drop deformation, calculations assuming the absence of any flow were also carried out, where Eqs. (6), (7) and (10-4) without hydrodynamic stress terms were used as governing equations and boundary conditions.

\subsection{Numerical procedure}

The finite element method for solving freeboundary problems is applied to the set of equations, Eqs. (5)-(12), describing the electric and flow fields around a drop and the drop shape.

The electric potential and velocity vectors are approximated by Lagrangian biquadratic polynomials $\phi$ defined over a mesh consisting of biquadratic quadrilateral elements.

$$
\begin{aligned}
& V(r, z)=\Sigma \phi_{i}(r, z) V_{i} \\
& v(r, z)=\Sigma \phi_{i}(r, z) v_{i}
\end{aligned}
$$

The dynamic pressure is approximated by linear basis functions $\psi$ which are discontinuous across each element.

$$
p(r, z)=\Sigma \psi_{i}(r, z) p_{i}
$$

Since the drop shape is itself a part of the solution, it should also be expressed by polinomials. The drop shape $f$ shown in Fig. 2 is approximated by one-dimensional Lagrangian quadratic basis functions $\Gamma$ as follows.

$$
f(\theta)=\Sigma \Gamma_{i}(\theta) f_{i}
$$


Table 2. Dimensionless Parameters

\begin{tabular}{llcl}
\hline & $\begin{array}{l}\text { Case 1 } \\
\text { continuous } \\
\text { phase: mixed oil } \\
\text { drop: silicone oil }\end{array}$ & Case 2 & $\begin{array}{l}\text { Case 3 } \\
\text { continuous } \\
\text { phase: silicone oil } \\
\text { drop: mixed oil }\end{array}$ \\
\hline$R$ & 0.016 & 0.283 & 62.9 \\
$S$ & 0.581 & 0.400 & 1.72 \\
$\kappa$ & 0.068 & 1.000 & 14.7 \\
$C a$ & 4.56 & 0.025 & 0.021 \\
$E_{0}{ }^{*}$ & $1 \sim 9$ & $1 \sim 6$ & $1 \sim 2.3$ \\
& $\Psi<0$ & $\Psi=0$ & $\Psi>0$ \\
& $R<S$ & $R<S$ & $R>S$ \\
\hline
\end{tabular}

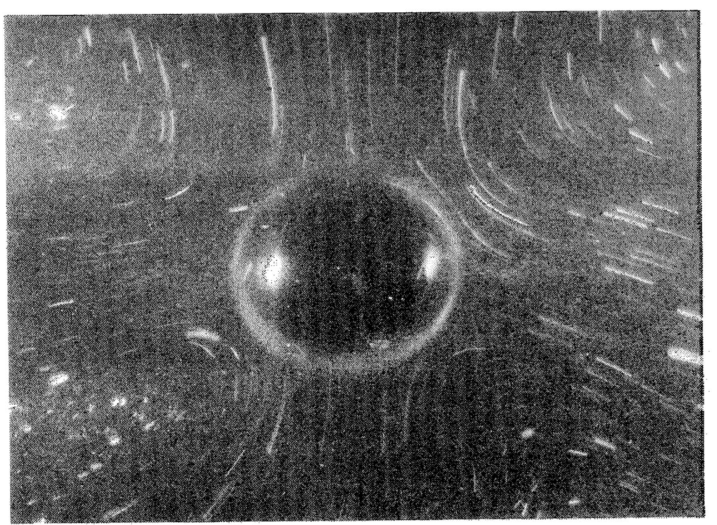

Fig. 4 Circulations outside a silicone oil drop under the influence of a uniform D.C. electric field which is applied vertically. The surrounding fluid is composed of castor oil and corn oil.

Applied voltage: 5,000 V. Exposure: $10 \mathrm{~s}$

Figure 3 shows the numerical mesh. Basically, the numbers of elements and nodes are 390 and 1,621 respectively. The outer boundary is set at five times the drop diameter far from the center of the drop. The discretization was confirmed to be enough to avoid any effect of mesh size on the solution.

The application of the Galerkin weighted residual method to Eqs. (5)-(12) and the solution approximations, Eq. (13), yield a set of nonlinear algebraic equations that are expressed by the following equations:

$$
\boldsymbol{R}(\boldsymbol{x} ; \boldsymbol{p})=0
$$

where $\boldsymbol{R}$ is a vector of residual equations, $\boldsymbol{x}$ is vector unknowns, i.e. electric potential, velocity vector, pressure, drop shape and so on, and $\boldsymbol{p}$ represents the dimensionless parameters listed in Table 2. We used the Newton method to solve Eq. (14).

\section{Results and Discussion}

In the present work, the focus has been placed on drop behaviors in the three cases shown in Table 2. Case 1 represents a system in which the drop and the continuous phase are composed of the silicone oil and the vegetable-oil mixture, respectively. Case 3 is opposite to Case 1. Case 2 represents an ideal fictional system which satisfies the condition for the drop shape being spherical,
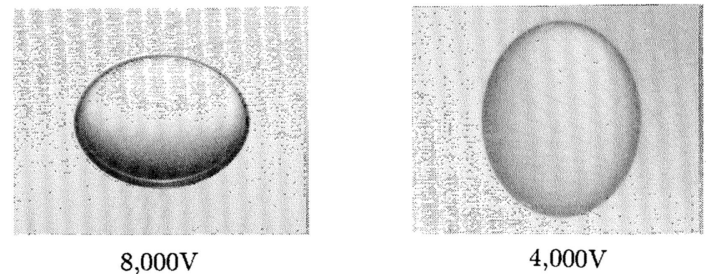

$4,000 \mathrm{~V}$

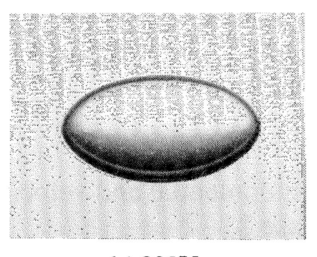

$14,000 \mathrm{~V}$

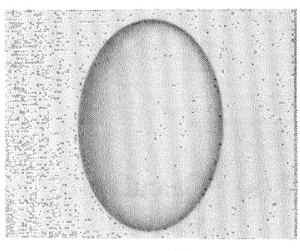

$4,500 \mathrm{~V}$

(a) Case 1

(b) Case 3

Fig. 5 Effect of an applied voltage on the drop shape in Cases 1 and 3

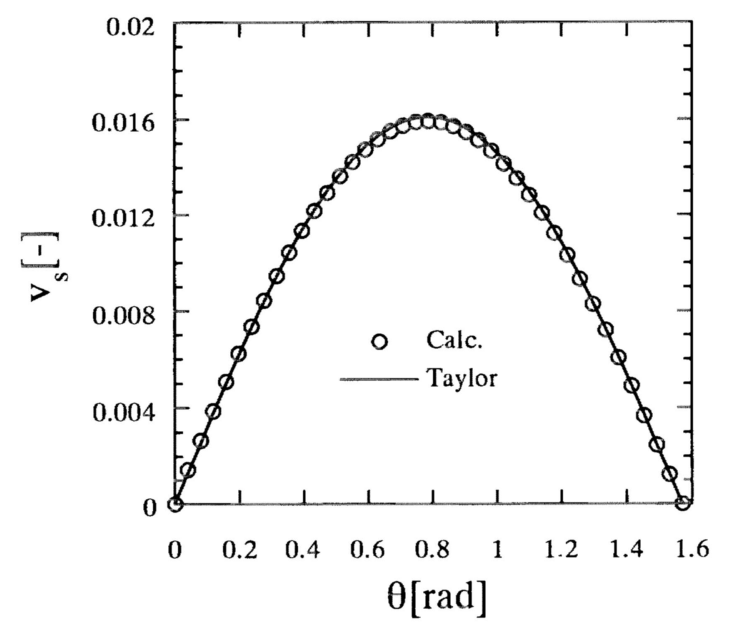

Fig. 6 Comparison between the calculated and Taylor's analytical solutions of the velocity on the surface of a drop in Case 2, when $E_{0}^{*}=1$

$\Psi=0$ in Eq. (1). The parameters listed in Table 2, such as $C a$ and $E_{0}{ }^{*}$, are made dimensionless with the volume equivalent diameter, $d_{e}=5 \mathrm{~mm}$, as the characteristic length.

Figure 4 shows a photograph demonstrating circulations outside a silicone oil drop in the vegetable-oil mixture (Case 1) at an applied voltage of 5,000 V. Stream lines which are almost symmetric about the equator are observed, and the flow direction from the pole to the equator was experimentally substantiated. These circulations are ascribable to a tangential stress at the drop surface caused by the electric field.

Figure 5 shows a series of photographs of drops subjected to various applied voltages. The drops deform more as the applied voltage increases in both Cases 1 and 3. Taylor ${ }^{7)}$ showed that drops deform into an oblate or a prolate spheroid when $\Psi$ is negative or positive 

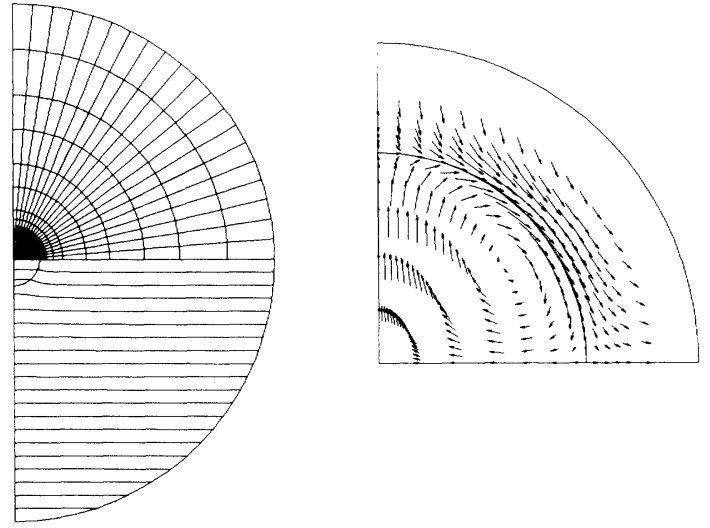

Fig. 7 (a) Calculated drop shape, equipotential and velocity vectors for $E_{0} *=5$, in Case 2
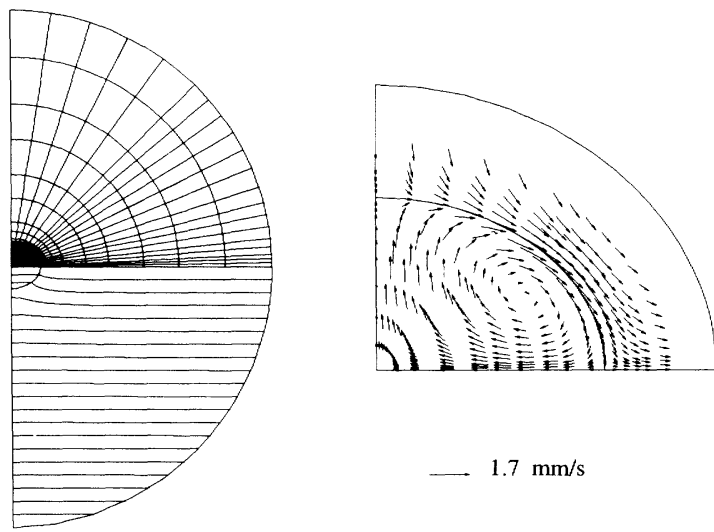

$\longrightarrow 1.7 \mathrm{~mm} / \mathrm{s}$

Fig. 7 (b) Calculated drop shape, equipotential and velocity vectors for $E_{0} *=5$, in Case 1
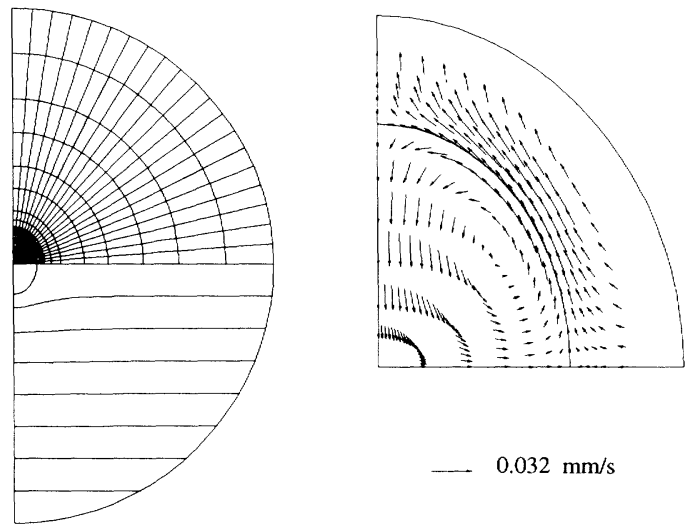

— $0.032 \mathrm{~mm} / \mathrm{s}$

Fig. 7 (c) Calculated drop shape, equipotential and velocity vectors for $E_{0} *=2$, in Case 3

respectively. In Cases 1 and 3, $\Psi$ is -0.212 and 0.964 , respectively. Apparently, the observed drop deformations agree with Taylor's criteria; that is, the drop becomes more oblate in Case 1 and more prolate in Case 3 with electric field strength. A silicone oil drop could be stable up to an applied voltage of $20,000 \mathrm{~V}$ in Case 1, although the drop deformed into a biconvex lens with a sharp edge above an applied voltage of $14,000 \mathrm{~V}$. On the other hand, a vegetable-oil drop could be stable up to an applied voltage of 5,000 V in Case 3, but it elongated to reach the electrodes at the higher voltage.

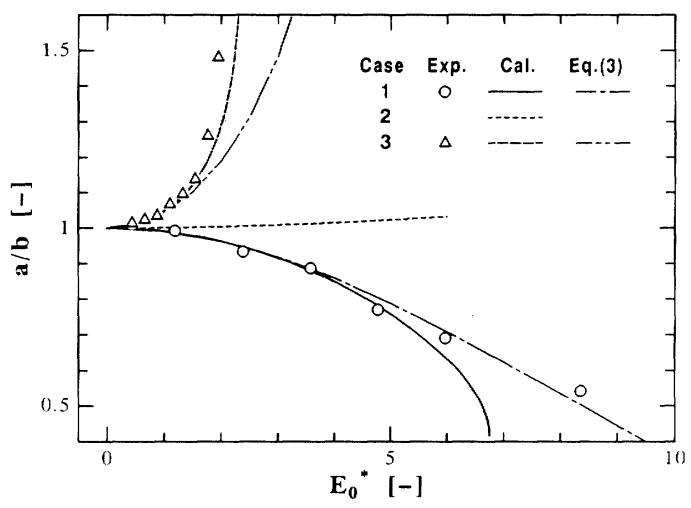

Fig. 8 Drop aspect ratio, $a / b$, as a function of the dimensionless strength of the applied electric field, $E_{0}{ }^{*}$

Next, we explain the calculated results by a mathematical model developed here. First, the calculated results in Case 2 are compared with the analytical solution given by Taylor ${ }^{7}$ to verify the validity of the model. He showed that a drop remains spherical under any electric field strength when $\Psi=0 . \Psi$ in Case 2 is 0.02 . Figure 6 shows a comparison between the calculated and Taylor's analytical results for velocity at the drop surface when $E_{0} *=1$. The validity of calculations in the present work is confirmed by the agreement of the two results.

Figure 7 shows the calculated equipotential lines, velocity vectors and drop shape in the three cases listed in Table 2, in addition to the finite element meshes used in calculations. In Case 2 shown in Fig. 7 (a), the drop remains spherical even at $E_{0}{ }^{*}=5$, and this also comfirms the validity of the present model. Fig. 7 (b) shows the calculated results in Case 1. It is found that the potential gradients are larger in the drop than in the continuous phase, that the drop shape is oblate, and that the flow direction is from the pole to the equator. These results agree with the experimental observations in Figs. 4 and 5 (a), while in Case 3, shown in Fig. 7 (c), the flow direction is from the equator to the pole and the drop shape is prolate. The calculated results for the flow direction and the drop shape in Cases 1 and 3 shown in Fig. 7 demonstrate theoretically Taylor's criteria. The maximum velocity in Cases 1 and 3 is $1.7 \mathrm{~mm} / \mathrm{s}$ and $0.032 \mathrm{~mm} / \mathrm{s}$ respectively, and it is expected that the hydrodynamic stresses in Case 1 affect the drop deformation much strongly than in Case 3.

Figure 8 shows a comparison between the calculated and experimental results for drop deformation under the influence of an electric field, where $a / b$ is the aspect ratio of the vertical axis to the horizontal axis of the drop. In Fig. 8, the results based on the expression for a drop deformation derived by Torza et al. ${ }^{8)}$, Eq. (3), are also shown. The calculated results are in good agreement with the experimental ones in both Cases 1 and 3, except at high electric field strength in Case 1. The possible reason for the discrepancy in Case 1 is that the drop deforms as a biconvex lens with a sharp edge at high $E_{0}{ }^{*}$, as shown in Fig. 5, and the present model is unable 


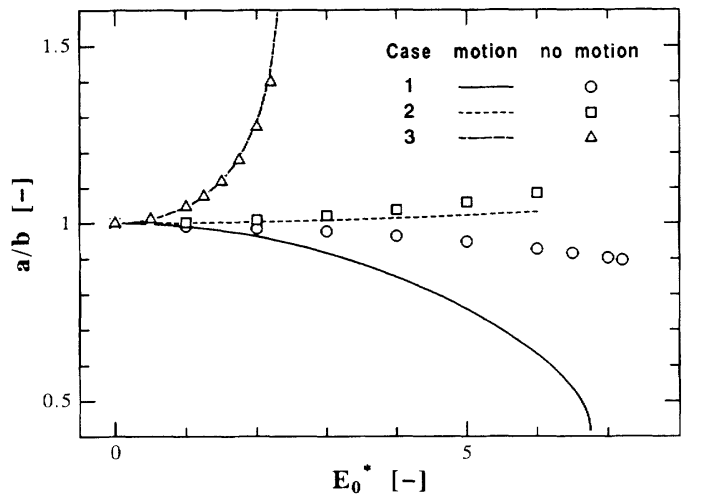

Fig. 9 Effect of the fluid flow on the drop deformation under the influence of the electric field

to deal with such deformation.

From the results, the drop becomes gradually more oblate in Case 1, and remarkably more prolate in Case 3 as $E_{0} *$ increases. The broken line corresponds to Case 2, and the drop shape remains almost spherical under any $E_{0}{ }^{*}$ as Taylor predicted. The results predicted by Eq. (3) are in good agreement with the numerical results by the present model at small $E_{0} *$ in both Cases 1 and 3, because this equation is the first-order approximation, which is valid only for a nearly spherical drop. Eq. (3) seems to predict well the experimental results even at high $E_{0} *$ in Case 1 , but, this may be a mere coincidence.

Figure 9 shows the effect of the flow field around the drop on drop deformation, that is, a comparison between the calculated results in Fig. 8 and the results obtained by neglecting the velocity field. From Fig. 9, no effect of the flow field on drop shape is found in Case 3. On the contrary, circulations around the drop have a great effect on drop deformation in Case 1, because the tangential stress induced by the electric field over the drop surface is much larger than in Case 3 and thus stronger circulations are generated around the drop, as shown in Fig. 7.

On the earth, circulations inside and outside drops, which enhance the heat and mass transfer rates between the drop/continuous phase in direct-contact heat exchangers or liquid extraction apparatus, can be induced easily by the influence of gravity. The application of an electric field as described here seems to be an additional technique for further strengthening the circulations. However, how about in space? If such apparatus were utilized under the micro-gravity, the other driving forces than gravity should be considered, such as the electic force described here. We would like to suggest that electric force is effective for moving dispersions and for inducing circulations inside and outside drops, and that the mathematical model developed here is useful for predicting and understanding these phenomena for the design of apparatus to be operated in space.

\section{Conclusions}

Theoretical and experimental studies of the behavior of a dielectric drop stationarily suspended in another dielecric liquid under a uniform electric field were carried out. A mathematical model that can exactly deal with the above problem was developed, and the calculated results with this method were in good agreement with the experimental observations.

\begin{tabular}{|c|c|c|}
\hline \multicolumn{3}{|c|}{ Nomenclature } \\
\hline$a$ & $=$ vertical length of drop & [m] \\
\hline$b$ & $=$ horizontal length of drop & [m] \\
\hline $\mathrm{Ca}$ & $=$ capillaly number $\left(=\rho_{1} v_{1}^{2} / \gamma d_{e}\right)$ & {$[-]$} \\
\hline$D_{\omega}$ & $=$ parameter defined by Eq. (4) & [-] \\
\hline$d_{e}$ & $=$ volume equivalent diameter & [m] \\
\hline$E$ & $=$ electric field & {$\left[\mathrm{V} \cdot \mathrm{m}^{-1}\right]$} \\
\hline$E_{0}$ & $=$ strength of electric field at outer boundary & {$\left[\mathrm{V} \cdot \mathrm{m}^{-1}\right]$} \\
\hline$E_{0}^{*}$ & $=E_{0} /\left(\gamma / 4 \pi \varepsilon_{1} d_{e}\right)^{1 / 2}$ & [-] \\
\hline$f$ & $=$ radial distance to drop surface & [m] \\
\hline$H$ & $=$ mean curvature & {$\left[\mathrm{m}^{-1}\right]$} \\
\hline$j$ & $=$ electric current density & {$\left[\mathrm{A} \cdot \mathrm{m}^{-2}\right]$} \\
\hline$n$ & $=$ normal unit vector & {$[-]$} \\
\hline$p$ & $=$ dynamic pressure & {$[\mathrm{Pa}]$} \\
\hline$R$ & $=\sigma_{2} / \sigma_{1}$ & {$[-]$} \\
\hline$r$ & $=$ radial distance in cylindrical coordinates & [m] \\
\hline$r_{0}$ & $=$ radius of outer boundary & [m] \\
\hline$S$ & $=\varepsilon_{2} / \varepsilon_{1}$ & {$[-]$} \\
\hline$t$ & $=$ tangential unit vector & {$[-]$} \\
\hline$u$ & $=$ radial component of velocity vector & {$\left[\mathrm{m} \cdot \mathrm{s}^{-1}\right]$} \\
\hline V & $=$ electric potential & [V] \\
\hline$v$ & $=$ velocity vector & {$\left[\mathrm{m} \cdot \mathrm{s}^{-1}\right]$} \\
\hline$v$ & $=$ axial component of velocity vector & {$\left[\mathrm{m} \cdot \mathrm{s}^{-1}\right]$} \\
\hline$z$ & $=$ axial distance in cylindrical coordinates & [m] \\
\hline$\Gamma$ & $=$ Lagrangian quadratic basis function & {$[-]$} \\
\hline$\gamma$ & $=$ interfacial tension & {$[\mathrm{mN} \cdot \mathrm{m}]$} \\
\hline$\varepsilon$ & $=$ permittivity & {$\left[\mathrm{F} \cdot \mathrm{m}^{-1}\right]$} \\
\hline$\varepsilon_{r}$ & $=$ dielectric constant & {$[-]$} \\
\hline$\theta$ & $=$ polar angle & [rad] \\
\hline$\kappa$ & $=\mu_{2} / \mu_{1}$ & {$[-]$} \\
\hline$\mu$ & $=$ viscosity & [Pa.s] \\
\hline$v$ & $=$ kinematic viscosity & {$\left[\mathrm{m}^{2} \cdot \mathrm{s}^{-1}\right]$} \\
\hline$\rho$ & $=$ density & {$\left[\mathrm{kg} \cdot \mathrm{m}^{-3}\right]$} \\
\hline$\sigma$ & $=$ electric conductivity & {$\left[\Omega^{-1} \cdot \mathrm{m}^{-1}\right]$} \\
\hline$\tau$ & $=$ stress tensor & {$[\mathrm{Pa}]$} \\
\hline$\tau^{E}$ & $=$ Maxwell's stress tensor & {$[\mathrm{Pa}]$} \\
\hline$\phi$ & $=$ Lagrangian biquadratic basis function & {$[-]$} \\
\hline$\Psi$ & $=$ parameter defined by Eq. (2) & {$[-]$} \\
\hline$\psi$ & $=$ linear basis function & {$[-]$} \\
\hline$\omega$ & $=$ frequency & {$[\mathrm{Hz}]$} \\
\hline
\end{tabular}

$<$ Subscripts $>$

$\begin{array}{ll}1 & =\text { continuous phase } \\ 2 & =\text { drop }\end{array}$

Literature cited

1) Chang, L.S., T.E. Carleson and J.C. Berg: Int. J. Heat Mass Transfer, 25, 1023-1030 (1982)

2) Chang, L.S. and J.C. Berg: Int. J. Heat Mass Transfer, 26, 823832 (1983)

3) Chang, L.S. and J.C. Berg: AIChE J., 31, 551-557 (1985)

4) Griffiths, S.K. and F.A. Morrison, Jr: Transactions of the ASME, 101, 484-488 (1979)

5) Karri, S.B.R. and V.K. Mathur: AIChE J., 34, 155-157 (1988)

6) Oliver, D.L.R., T.E. Carleson and J.N. Chung: Int. J. Heat Mass Transfer, 28, 1005-1009 (1985)

7) Taylor, G.: Proc. Roy. Soc., A291, 159-166 (1966)

8) Torza, S., R.G. Cox and S.G.Mason: Phil. Trans. Roy. Soc., 269A, 295-319 (1971) 